\title{
DIE JUNGFRAU
}

\author{
SYMBOLIK EINES BERGES
}

\section{Eduard Gerber}

Die Vielfalt der Oberflächenformen der Erde beschäftigt unsern Geist auf grundsätzlich zwei Arten. Wir können versuchen, die Formen so zu beschreiben, zu ordnen und zu erklären «wie sie sind», oder wir können das Erlebnis festhalten und zu verstehen versuchen, wie diese Formen auf uns einwirken.

Der ersten Betrachtungsart befleißigt sich die Naturwissenschaft, für welche die Oberflächenformen ein vom menschlichen Empfinden unabhängiges Objekt sind, weshalb ihre Methode auch objetivierend genannt wird. Bei der zweiten Betrachtungsweise wird umgekehrt gerade der Einfluß auf das einzelne Subjekt beschrieben, was meist mit künstlerischen Methoden nach ästhetischen Gesichtspunkten geschieht und dann subjektiv genannt wird. Es kann dieser Einfluß aber objektiv psychologisch untersucht werden, wobei das primäre Objekt dann allerdings der Mensch ist.

Die gleichen Oberflächenformen dringen also offensichtlich durch verschiedene Sphären in unser Bewußtsein ein, beeinflussen uns vielleicht auch noch rein unterbewußt. Doch können wir, solange es unterbewußt ist, darüber nichts Bestimmtes aussagen, es höchstens ahnen. Und zwar durchdringen sich subjektive und objektive Sphären auch dort, wo wir uns anscheinend rein wissenschaftlich-objektiv verhalten. Zum Teil scheinen sich objektive und subjektive Aussagen auszuschließen, weil sie sich widersprechen, trotzdem beide «wahr» sein können, wie z. B. eine wissenschaftliche Zeichnung und ein künstlerisches Bild desselben Berges. Allein schon die grundlegende Tatsache, $\mathrm{da} \beta$ wir eine Form nur beschreiben können, wenn wir sie nicht als Chaos, sondern als wohlabgegrenzte Gestalt sehen und aus der doch unfaßbaren Mannigfaltigkeit, ob wir wollen oder nicht, einzelne Gestalten bevorzugen und herausheben, ist bei allem objektiven Bemühen eminent subjektiv.

$\mathrm{Zu}$ den bevorzugten Gestalten der Schweizeralpen gehört die Jungfrau. Es gibt wohl viele solcher Bergformen, die einer bestimmten Gegend das Gepräge geben oder einen besondern Akzent setzen, so der Glärnisch dem Zürichsee, die Bernina dem Oberengadin, der Säntis der Ostschweiz. Zwei Gipfel aber besitzen nicht nur regionalen, sondern nationalen, ja internationalen Ruf: die Jungfrau und das Matterhorn. Für eilige Reisen ausländischer Gesellschaften gilt der Besuch von Interlaken mit einem raschen Blick auf die Jungfrau (schönes Wetter vorausgesetzt!) als unerläßlich und daß das Matterhorn vielen aus den USA anfliegenden Passagieren ein Begriff ist, beweist das Swissairbüchlein, in welchem es heißt: «When you land at Geneva Airport, you are on Swiss soil in the heart of Europe. You won't see the Matterhorn from the airport.»

Eine Konfrontation der zwei Berggestalten ist aufschlußreich. Das Matterhorn ist eine scharfkantige, vierseitige Felspyramide, isoliert, auf einem breiten, gletschertragenden und gletscherüberschliffenen Sockel. Es ist morphologisch der Typus eines Karlings, der Erosionsrest liegender Falten der Dentblanchedecke, von allen Seiten glazial unterschliffen, intensiver Frostverwitterung ausgesetzt. Was uns in höchstes Erstaunen, ja in Erregung bringt, ist die Größe und Kühnheit der Form, wohl auch unterbewußt der Symbolcharakter der Männlichkeit. Wir sehen das Horn allein, ohne Rivalen, hochaufstrebend. Die Distanz ist wichtig. Aus der Ferne, als Gipfel unter Gipfeln, ist die Wirkung gering. Nicht jede Seite des Matterhorns ist gleichwertig, doch von allen ist es imponierend. Unübertrefflich ist die Zermatterseite. Die Ansicht von Breuil ist lange nicht so günstig. Beide Orte, aber nur diese, haben durch das Matterhorn einen gewaltigen wirtschaftlichen Aufschwung genommen. Es hat einen Rivalen, den Muztagh Tower, im Karakorum, $7273 \mathrm{~m}$, «nach den Umrissen des Matterhorns aufgebaut, aber ungeheuer viel größer», jedoch ohne die Alpweiden und ohne Zermatt.

Die Jungfrau ist ein Glied der Randkette im Aaremassiv, in welches der Sedimentmantel in mächtigen Keilen hineingefaltet ist. Im Steilabsturz zur Scheidegg lagert im Gipfel Gneis auf gestuften Kalkwänden. Auf der Nordseite liegen östlich der Guggigletscher und Kühlauenengletscher; zentral zwischen zwei Vorgipfeln, dem Schneehorn 
und Silberhorn, unter einer hochaufstrebenden bogenförmigen Karwand der stark zerklüftete Gießengletscher. Auf der Westseite bildet der Schwarzmönch den Vorbau, der bis ins hintere Lauterbrunnental auf $900 \mathrm{~m}$ in steilen Felswänden abstürzt. Im Südwesten ioigt der gletschererfüllte Kessel des Rottales, aus dem der Giptei in steilen Wänden emporstrebt. Im Süden und Südosten reicht der Jungfraufirn, einer der Arme, die sich im Konkordiaplatz treffen und dann den Aletschgletscher bilden, bis unter die ihn nur noch wenig überhöhenden Gipfelfelsen hinauf. Im Osten bildet das Jungfraujoch die Verbindung zum Mönch. Die verschiedenen Gletscher liegen alle in weitgespannten Mulden und bieten vielleicht ein Bild dar wie das Matterhorn in später Eiszeit.

Die Jungfrau wirkt im Gegensatz zum Matterhorn häufig als Kettenglied und wird meist in einem Atemzug mit Mönch und Eiger genannt. Sie hat eine ausgesprochene Schauseite, weit weniger günstige Seitenansichten und auf der Rückseite keine besonderen Merkmale. Auf der Schauseite aber ist sie ausgesprochen schön. Sie ist nach den klassischen Regeln der Ästhetik aufgebaut, als Großform ein gleichschenkliges Dreieck und doch nicht starr symmetrisch. Der Gipfelaufbau, das Haupt, etwas zurückversetzt, wird flankiert von zwei Vorbauten, beide wieder von Dreiecksform, wobei der eine Schenkel des linken Vorbaues diagonal zur Mitte der Basis herabzieht. Als mächtige Architektur muß die Jungfrau auch Ferdinand Hodler erschienen sein, der sie mehrmals malte, dem Künstler, den PaUl Niggli den «großen Verkünder der wieder erwachten Sinnes für Tektonik» genannt hat und der selbst gesagt hat: «Sehen: das ist die Verhältnismäßigkeit aller Erscheinungen erkennen.»

Die ganze Form gleicht einem großen Stuhl mit breitausladenden Lehnen, einem Götterthron, ja, wir können weitergehen und diese machtvolle Gestalt personifizieren zur hoheitsvoll thronenden Jungfrau von blendendem, unberührtem Weiß und mächtigem Schoß, zum Symbol der anmutsvollen Weiblichkeit. Als Jungckfraw ist sie schon 1577 urkundlich belegt und mag ihren Namen von den Augustinermönchen von Interlaken erhalten haben. Die nüchternere Deutung, daß der Name von einer Alp der Klosterfrauen von Interlaken abzuleiten sei, ist weniger wahrscheinlich, tut auch dem heutigen Symbolcharakter dieses Berges keinen Abbruch.

Die Wirkung, die diese wundervolle Berggestalt ausstrahlt, ist weitreichend. Wie im Theater aus dem ersten Rang, kann sie von der Wengernalp betrachtet werden, getrennt nur durch das tiefeingeschnittene Trümmeltal, in das auch an heißen Sommertagen Lawinen niederdonnern. Dort ist der Berg noch mit allen Detail erkennbar, noch nicht «stumm» wie aus weiter Ferne. Berühmt ist die Ansicht von Wengen, wo die Jungfrau, wie das Bild zeigt, über weite Wies- und Alpflächen und über Wälder emporsteigt. Weniger günstig ist die Ansicht von Mürren, jenseits des Lauterbrunnentales, wo die dunkeln Felsmassen der Westseite vorherrschen. Wohl eine der schönsten Ansichten als blendend weiße Berggestalt bietet von jenseits des Lauterbrunnentales die Gegend von Isenfluh. Eine ganze Reihe von Aussichtspunkten, bei denen der Blick auf die Jungfrau das zentrale Erlebnis ist, wurden ausgebaut, so die Schynige Platte, zu der eine Zahnradbahn führt, der Harder bei Interlaken mit einer Drahtseilbahn, das Niederhorn ob Beatenberg mit einer Sesselbahn.

Der seit dem Beginn der Alpenreisen berühmteste Ort ist aber unzweifelhaft Interlaken mit dem Höhenweg und der Höhenmatte, dem alten Gerichts- und Landsgemeindeplatz der Oberländer. Links und rechts des Lütschinentales versperren Voralpenketten den Blick auf das Hochgebirge, aber mitten über der Talsohle leuchtet als der einzige sichtbare Gipfel die Jungfrau in ihrer unvergleichlichen Dreiecksform, blendend weiß zwischen den dunkelgrünen, von Fels durchzogenen Waldhängen.

Aber die Ausstrahlungen reichen weiter, weit über das Voralpenland hinaus. Zwar ist es nur selten die Jungfrau allein, meist sieht man einen größern Teil des Kranzes der Schneeberge. Immer jedoch dominiert die Jungfrau. Denken wir an die Aussichtspunkte des Emmentales - die Moosegg z. B. - an den Gurten bei Bern oder an Bern 


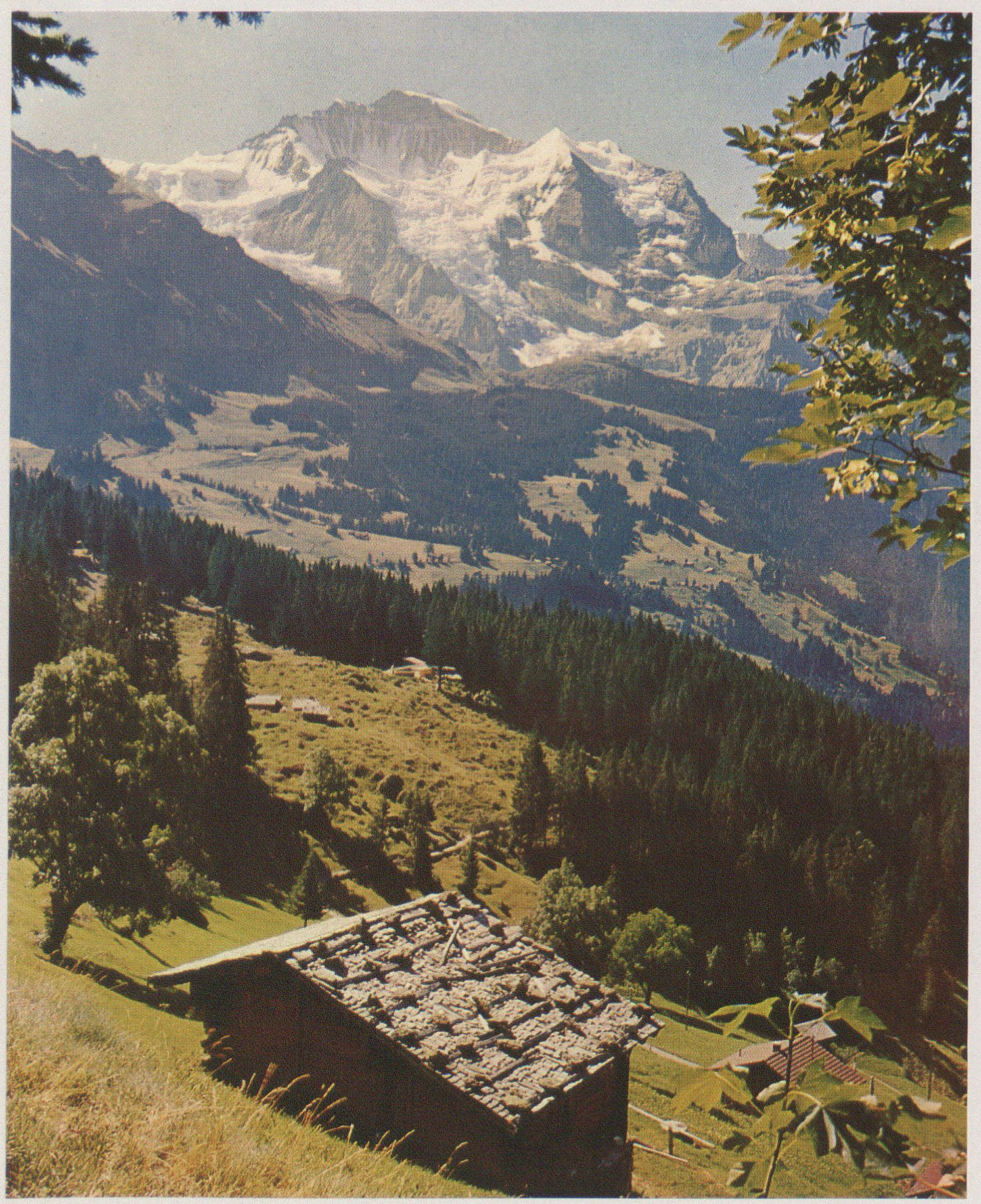

Typische Berner Oberländer Landschaft mit Blick auf die Jungfrau 
selbst, etwa an die Bundesterrasse, oder an die Engehalbinsel, mit der Stadt und dem Münsterturm im Vordergrund, wenn die Berge im Mittagsglanz leuchten oder im Alpenglühen stehen - wie viele sind dann gebannt von diesem Anblick!

Noch weiter! Wir gedenken des Blickes vom Jura über das Mittelland mit seinem Mosaik von Äckern, Wiesen und Wäldern vom Chaumont, Chasseral, dem Weißenstein zum Kranz der Alpen mit der zentralen Gestalt der Jungfrau. Ja, über den Jura hinweg vom Gebweiler Belchen in den Vogesen ist die Jungfrau zu sehen.

Bei großen Teilen des Alpenpanoramas, das vom Mittelland aus gesehen werden kann, werden die meisten Betrachter nur ganze Gebirgsgruppen benennen können, häufig sind es nur einfach «die Alpen». Die Berneralpen mit ihren ausgeprägt individuell profilierten Gestalten machen indessen eine Ausnahme und wenn auch die Namenkenntnis der Betrachter als sehr gering zu veranschlagen ist, so kennen doch die meisten den Namen der Jungfrau.

Die Wirkungen dieses Berges sind nicht nur weitreichend, sondern auch mannigfaltiger Art. Sie sind auch wirtschaftlich nicht zu unterschätzen, wenn wir an den Fremdenverkehr denken. Dies findet bei manchem Hotel Ausdruck, das Jungfrau oder Jungfraublick heißt. Über dieses noch in Franken und Besucherzahlen Meßbare hinaus reicht die Bedeutung ins Unmeßbare. Durch Jahre trugen die eidgenössischen Abstimmungsanschläge als zierende Kopfzeichnung die Jungfrau. Denn die Jungfrau, die zu gewissen Tageszeiten ein Schattenkreuz trägt, ist weit über das Ästhetische und über das Bergerlebnis des Einzelnen hinaus zu einem eidgenössischen Symbol geworden.

So zeigt das Beispiel der Jungfrau, daß der Einfluß eines Berges weit über seinen Standort ausstrahlen kann und die geographische, die landschaftliche Bedeutung einer solchen Gestalt nur erfaßt wird, wenn neben die rein naturwissenschaftlich-objektive Beschreibung die ästhetisch-psychologische Würdigung tritt.

Michel, H.: Die Jungfrau, Berner Heimatbücher Nr. 25 Bern - Lucerna, R.: Matterhorn. Peterm. geogr. Mitteilungen 22, 1936. - Niggli, P.: Schulung und Naturerkenntnis. Zürich 1945. - Hartog, J. M. Muztag Tower. Die Alpen 1957 - Boss, V. Lütschinentäler. Berner Wanderbuch 6. Bern 1959.

\title{
ZUR BEWÄSSERUNG DER POEBENE
}

\author{
Walter Nelz
}

\section{EIN LEITUN G}

Bewässerungsanlagen sind wichtige Kulturlandschaftselemente in weiten und geschichtlich bedeutungsvollen Gebieten der Erde. Geographen und Geschichtsforscher kennzeichneten die orientalischen Kulturen vom Nil bis zum Hoangho als Fluß-und Bewässerungskulturen (potamisch) und stellten ihnen die europäischen Meerkulturen (als thalassisch und ozeanisch) gegenüber, wie z. B. ERnst KAPP. KARL MARX und andere verstanden als grundlegendes Element der «asiatischen» Produktionsweise den großen Bewässerungslandbau, der in der Sumpfreiskultur eindrücklich in die Erscheinung tritt.

Nach diesen Schriftstellern entstand und entwickelte sich anfangs die territorialstaatliche Zivilisation in den Flußtälern des Orients. Die Nutzung des Wassers großer Ströme erforderte soziale Kooperation und war deshalb ein organisierender, staatsbildender Faktor ersten Ranges. In unserer Zeit aktualisieren sich die alten Beziehungen zwischen Morgen- und Abendland auf neue Weise. Die orientalischen Kulturen beanspruchen deshalb unsere Aufmerksamkeit in steigendem Maße. Es sei in diesem Zusammenhang nur auf die Schriften des Soziologen K. A WitTFOGEL verwiesen. In «Wirtschaft und Gesellschaft Chinas» (Leipzig 1931) untersuchte er sowohl die Funktion der Bewässerung im landwirtschaftlichen Produktionsprozeß Chinas, als 